\title{
Wiederaufleben der deutsch-französischen Partnerschaft? Eine Bestandsaufnahme im doppelten Wahljahr 2017
}

Bei einem Blick auf die europäischen Reformbestrebungen der letzten Jahre stellt sich die Frage: Wo war in dieser Zeit Frankreich? Wo sind die Impulse geblieben, die die Republik seit Anbeginn der europäischen Integration stets gegeben hat, die sich jahrzehntelang als der politische Kopf dieses Zusammenschlusses gesehen hat? Ausgehend von den Gründen für die französische Passivität werden die Pläne des Präsidenten Emmanuel Macron in der europäischen Finanzpolitik, der Ausrichtung der EU, der Sozial- sowie der Verteidigungspolitik analysiert.

\section{Die Abwesenheit Frankreichs}

Frankreich hat sich eigentlich nie aus der ersten Reihe der Europäischen Union verabschiedet. Während der Amtszeit der zwei letzten Präsidenten, Nicolas Sarkozy (2007-2012) und François Hollande (2012-2017) konnte es jedoch nur eingeschränkt die Politik der EU mitgestalten. Im Wesentlichen gab Deutschland die Leitlinien vor, französische Gegenvorschläge wurden abgeschmettert, oft genug wurde nur eine Abmilderung des deutschen Kurses erreicht. Das zeigte sich zum Beispiel bei der europäischen Schuldenkrise, in der Berlin einen harten Kurs gegenüber den Ländern fuhr, die die Solidarität der anderen Mitgliedsstaaten in Anspruch nahmen, um den rasant ansteigenden Zinssätzen auf ihren Schuldpapieren Herr zu werden.

Die Übernahme von Garantien durch die EU war an Reformmaßnahmen gekoppelt, die vor allem eine Reduzierung der Ausgaben im Blick hatten. Die sozialen Härten, die dieser als „Austeritätspolitik“ bezeichnete Weg mit sich brachte, stellten eine erhebliche Belastungsprobe für den Zusammenhalt der EU dar. Frankreich legt traditionell großen Wert auf eine umfangreiche soziale Absicherung seiner Bürger, und setzt eher auf eine nachfrageorientierte Politik. Zudem sind der Republik die Beziehungen zu den unmittelbaren Nachbarländern Spanien und Italien, sowie generell zu den Mittelmeeranrainerstaaten, außerordentlich wichtig. Nicht zufällig war eines der letzten großen europäischen Initiativen Frankreichs die Bildung einer Mittelmeerunion, die EU-Staaten mit anderen Mittelmeeranrainern verband (Gougeon, 2012, S. 22f.). Wurde unter Sarkozy noch hinter den Kulissen mit dem deutschen Partner gerungen, scheute der Sozialist Hollande auch nicht eine offenere Konfrontation, indem er sich öffentlichkeitswirksam mit den Regierungschefs der südlichen Mitgliedsstaaten gegen weitere Austeritätsmaßnahmen positionierte, allerdings ohne Erfolg. 
Warum reichte der französische Einfluss, der noch bei den Verträgen von Nizza (2003), dem Projekt einer europäischen Verfassung (2004-2005) und letztlich beim Lissabon-Vertrag (2009) deutlich zu spüren war, nicht mehr zur Durchsetzung einer anderen europäischen Finanzpolitik? Die Gründe sind in der anhaltenden Schwäche der französischen Wirtschaft seit Beginn der globalen Finanzkrise sowie einer gesellschaftlichen Spaltung über Europafragen zu finden.

Wenige EU-Staaten wurden von der Finanzkrise, die 2007 begann und in den Folgejahren ihre volle Wucht entfaltete, verschont. Ein Land nach dem anderen sah sich gezwungen Schulden in größerem Umfang aufzunehmen, um die laufenden Staatsausgaben während dieser Konjunkturschwäche zu finanzieren. Deutschland war da keine Ausnahme, der Bundeshaushalt erholte sich jedoch relativ schnell. Reformen zur Steigerung der deutschen Wettbewerbsfähigkeit waren bereits im Vorfeld durchgeführt worden, und das Modell der exportorientierten Wirtschaft profitierte von der sich erholenden globalen Ökonomie überdurchschnittlich gut.

Frankreich dagegen hatte zu Beginn der Finanzkrise ein verlorenes Jahrzehnt hinter sich. Zwar erkannten die verschiedenen Regierungen, dass das französische Wirtschaftsmodell nicht mehr so gut funktionierte wie in früheren Jahrzehnten, die Staatsausgaben aus dem Ruder liefen und sich Reformbedarf anstaute, doch über den Ausweg waren sie sich mit maßgeblichen gesellschaftlichen Gruppen uneins (Lasserre, 2012, S. 152). Der damalige Trend zum Neoliberalismus war in Frankreich unpopulär, und so recht vertrug er sich auch nicht mit dem Ansatz einer interventionistischen Staatsführung, wie man sie in Paris stets pflegte. Der Abbau sozialer Leistungen stand außer Frage, auch eine Flexibilisierung des Arbeitsmarktes zuungunsten der Arbeitnehmerschaft stieß auf erhebliche Widerstände, sowohl im Parlament als auch in der Bevölkerung. Der Versuch, 2006 die Probezeit für junge Arbeitnehmer auf zwei Jahre auszuweiten (der sog. „Contrat Première Embauche“), löste einen derartigen Proteststurm aus, dass das entsprechende Gesetz zurückgezogen wurde. Ebenso fruchtete ein dutzende Milliarden Euro schweres staatliches Investitionsprogramm unter Sarkozy nicht, eher moderate Reformbemühungen unter Präsident Hollande (u.a. die Einführung neuer Steuerkredite für Unternehmen) zeigten nur langsam Wirkung.

Eine Folge dieses wirtschaftspolitischen Stillstandes war die regelmäßige Verletzung der Maastricht-Kriterien, die eine maximale jährliche Neuverschuldung von maximal 3\% des Bruttoinlandsprodukts (BIP) vorsieht. Seit 2008 wurden diese Kriterien nicht mehr erfüllt. Zudem stieg die Gesamtverschuldung von 63,2\% des BIP im Jahr 2007 auf über 95\% im Jahr $2016^{1}$ (Eurostat, 2017, S. 1). Unter diesen Umständen glich die Überprüfung des Staatshaushaltes durch die Europäische Kommission, die gemäß den Konvergenzkriterien erfolgt, einem wiederkehrenden Gang nach Canossa. Selbst die relativ laxe Interpretation der Kriterien durch die Kommission unter Vorsitz von Jean-Claude Juncker, die vor der Verhängung vorgesehener Strafzahlungen für ein so großes Land wie Frankreich zurückschreckte, brachte nur eine begrenzte Entspannung mit sich. Entscheidend ist nämlich die deutsche Wahrnehmung: solange Frankreich nicht zumindest seine Neuverschuldung in den Griff bekommt, wird Paris

${ }^{1}$ Während die Bundesrepublik Deutschland lediglich 2009 (3,2\% Defizit) und 2010 (4,2\% Defizit) die Kriterien verletzte, schwankten die jährlichen französischen Defizite in der Periode 20082016 zwischen 3,2\% und 7,2\% (Eurostat, 2017, S. 1). 
nicht als ernstzunehmender Partner in finanziellen Belangen betrachtet. In der Tat fällt es schwer dem Kurs einer Regierung zu folgen, die mit dem eingeschlagenen Weg permanent die eingegangenen Verpflichtungen nicht einhält.

Der finanzielle Notstand fachte auch die innerfranzösische Problematik an. In Zeiten knapper Kassen werden gesellschaftliche Konflikte, die durch eine großzügige Verteilungspolitik überdeckt wurden, akuter. Der ohnehin bestehende Dissens über die beste Möglichkeit, Frankreich in die Zukunft zu führen, weitete sich aus. Die allgemein als mutlos wahrgenommene Politik von François Hollande wurde von verschiedenen Seiten unter Beschuss genommen. Die Rechtsextremen unter Marine Le Pen propagierten einen nationalistischen Protektionismus, der in einem Rückzug aus den europäischen Institutionen münden sollte. Die Konservativen entwickelten im Laufe des Wahlkampfs 2016-2017 ein neoliberales Konzept, das den massiven Abbau von Stellen im öffentlichen Dienst und reduzierten Ausgaben im Gesundheitssektor vorsah. Auf der linksextremen Seite wurde eine Neuverhandlung der europäischen Verträge gefordert, die einen neuen öffentlichen Investitionsschub ermöglichen sollte. Später kamen noch die Forderungen von Emmanuel Macron dazu, der einen sozialliberalen Reformweg propagierte (Vgl. Wernert, 2017, S. 11ff.).

Im Strudel der innerfranzösischen Konflikte gelang es François Hollande nicht, der Stimme seiner Regierung auf europäischer Ebene Gehör zu verschaffen, zumal die Finanzen seines Landes weiterhin problematisch waren (Schmitt, 2017, S. 40f.). Die deutsch-französische Partnerschaft mutierte zu einer dysfunktionalen Beziehung, bei der Deutschland den Ton angab und Frankreich mühsam versuchte, Korrekturen einzubringen. Die Stellung der Bundesrepublik, die auch als hegemonial bezeichnet wurde, war auch für die deutsche Regierung keineswegs erwünscht oder gar angenehm. Der Schatten eines übermächtigen Deutschlands in Europa rief böse Erinnerungen wach, und sowohl das politische Personal, als auch die Wirtschaftskraft der Bundesrepublik waren nicht einer alleinigen Führungsrolle in der EU gewachsen.

\section{Neuanfang unter Macron}

Die obige Bestandsaufnahme lässt erahnen, mit wieviel Erleichterung die Wahl Emmanuel Macrons zum Präsidenten und seiner Partei „La République en marche!“ vom deutschen Partner aufgenommen wurde. Einerseits war damit in Frankreich ein vorübergehender Konsens über den zu gehenden Reformweg hergestellt, andererseits meldet der Neuankömmling in der europäischen Politik Gestaltungsansprüche an, wie sie lange vermisst wurden.

Die Wahl Macrons wurde in Deutschland zunächst als Abwehr des größten anzunehmenden Unfalls, der Wahl der rechtsextremen Marine Le Pen, empfunden. Wäre Le Pen gewählt worden, hätte dies womöglich zur Implosion der Europäischen Union geführt, im allerbesten Fall wäre jegliche EU-Reform über fünf Jahre hinweg unmöglich gewesen. Als Gründungsmitglied und zweitgrößte Volkswirtschaft der Eurozone ist Frankreich im europäischen Gefüge schlicht unentbehrlich.

Einige Parameter haben sich rechtzeitig zum Amtsbeginn von Macron zugunsten einer stärkeren Rolle Frankreichs geändert. Der Frage des Haushaltsdefizits könnte 
schon durch die begrenzten Bemühungen Hollandes die Schärfe genommen werden. Gegenwärtig rechnet das Finanzministerium mit einem Defizit von 2,8\% für das Haushaltsjahr 2017. Die symbolisch und rechtlich wichtige Grenze der 3\% wäre damit unterschritten, sodass ein wesentlicher Kritikpunkt der deutschen Seite aus dem Weg geräumt wäre. Die Stabilisierung der finanziellen Lage erlaubt Macron auch etwas Spielraum bei der Ausgestaltung seiner innenpolitischen Vorhaben (u.a. bei der Senkung der Unternehmenssteuer auf 25\%), sodass ihm nicht von Anfang an die Hände gebunden sind. Zurzeit profitiert die französische Wirtschaft von einem niedrigen Ölpreis und einer positiven europäischen Dynamik, das Defizit könnte unter ungünstigeren Umständen leicht wieder die 3\%-Grenze durchbrechen. Der Rechnungshof hat Ende Juni 2017 bereits angemahnt, dass die Prognose von 2,8\% Defizit falsch sei und eher 3,2\% entspräche (Cour des comptes, 2017, S. 48ff.).

Eine der Prioritäten Macrons ist es, ein Arbeitsmarktgesetz zu verabschieden, das die zentralen Probleme der französischen Wirtschaft angeht: ein erstarrter Arbeitsmarkt, der besonders zuungunsten der jüngeren Generationen funktioniert, eine von schweren Zerwürfnissen geprägte soziale Partnerschaft zwischen Arbeitnehmer und -geber, ausufernde Kosten des Staatsapparats und der Sozialversicherung. Erklärtes Ziel im Wahlkampf Macrons war es, durch diese Reformen nicht nur innenpolitische Probleme zu lösen, sondern dadurch auch wieder internationale Glaubwürdigkeit zu erlangen und in den europäischen Fragen an prominenter Stelle mitzugestalten (Uterwedde, 2017, S. 38). Münden die Reformen in entsprechende Gesetze und tragen auch ihre Früchte, besteht Hoffnung auf eine dauerhafte Erholungen der französischen Wirtschaft und Finanzen. Der Weg wäre dann frei für eine starke Position in den Verhandlungen zu den angedachten europäischen Projekten.

\section{Europäische Finanzpolitik}

Eine erste Initiative Frankreichs betrifft die Gestaltung der europäischen Finanzpolitik. Macron wünscht sich einen europäischen Finanzminister, der für eine wachsende Konvergenz in der EU sorgt. Unter diesem Schlagwort kann man sich allerlei Inhalte vorstellen. Grundsätzlich ist Deutschland ebenfalls für die Schaffung einer solchen Position, denkt dabei aber an einen obersten Finanzaufseher, der abseits der Europäischen Kommission und ihren politischen Erwägungen (man denke an die ,politische Kommission“, die Jean-Claude Juncker anführt) die Einhaltung der Maastricht-Kriterien strikt und unabhängig überwacht. Die Einhaltung der Schuldenquote ist für viele Mitgliedsstaaten auch mittelfristig nicht zu erreichen, im Fokus stünde die Neuverschuldung. Die seit Beginn der Schuldenkrise immer wieder von der CDU erhobene Forderung, strikt auf die Einhaltung der Verträge zu pochen, würde damit institutionell verankert werden. Von einer SPD-geführten Regierung wäre ein deutlich milderer Tonfall gegenüber Mitgliedsstaaten zu erwarten, die in finanzielle Nöte geraten sind, auch dürften die Maastricht-Kriterien nicht mehr als unverrückbares Dogma verteidigt werden.

Mehr Flexibilität wäre auch im französischen Sinne, denn hier zeigt sich wieder die traditionell nachfrageorientierte Ausrichtung der Republik. Aus den Äußerungen im Wahlkampf ist zu entnehmen, dass mit dem europäischen Finanzminister eher die 
Grundlage für eine investitionsfähige und -freudige EU geschaffen werden soll. So darf auch die Forderung eines eigenen Budgets für die EU verstanden werden, dessen Einnahmen sich aus einer Finanztransaktionssteuer oder einer $\mathrm{CO}_{2}$-Steuer speisen könnte. Eine grundsätzliche Inkompatibilität besteht nicht, wenn ein Kompromiss hinsichtlich der Einhaltung der (überarbeiteten?) Maastricht-Kriterien erzielt werden könnte, und Deutschland sich zu mehr finanziellem Engagement verpflichten würde, wozu es momentan unter dem Eindruck des Le Pen-Schocks bereit sein dürfte (Berger, 2017, S. 1). Grundsätzlich liegt die Macron-Regierung aber näher an den Vorstellungen der SPD, als an denen der Union. Im SPD-Regierungsprogramm finden sich Forderungen nach europäischen Investitionen, einem „Europa der Solidarität“ und einem ,sozialen Europa“, die sich mit Macrons Plänen weitgehend decken (SPD, 2017, S. 74ff.). Im Unionsprogramm wiederum ist etwas vager von einem europäischen Wohlstandsraum die Rede, der die oben genannten Aspekte zumindest nicht ausschließt (CDU, CSU, 2017, S. 57). Auffällig ist, dass in beiden Regierungsprogrammen die Kooperation mit Frankreich als Schlüsselelement europäischer Reformen gesehen wird (CDU, CSU, 2017, S. 58; SPD, 2017, S. 74).

\section{Ein Europa mit klaren Wertvorstellungen}

Ein Finanzminister würde die europäische Integration vertiefen, abseits der Mechanismen zur Bewältigung der Finanzkrise wäre das der erste große Wurf seit dem Lissabon-Vertrag vor nunmehr acht Jahren. Das wirft auch die Frage nach der Wichtigkeit der Grundlagen der EU auf. Das Zurückdrehen demokratischer Kontrollinstanzen und die Einschränkung der Pressefreiheit in Ungarn seit dem Amtsantritt Viktor Orbans, Wortführer einer Wende hin zu einer „illiberalen Demokratie“, sowie in Polen unter der Ägide der PiS-Regierung, wirft im restlichen EU ein Dilemma auf. Wie soll und kann auf diese Entwicklungen reagiert werden?

Berlin vertritt eine eher leise Linie, die zwar in persönlichen Gesprächen den Unmut über die Verletzung über europäische Werte kundtut, sich öffentlich aber zurückhält. Die Bundesregierung legt besonderen Wert auf die Beziehungen zu Mittel- und Osteuropa, und sieht sich in einer Mittlerrolle zwischen dieser Region und Westeuropa. Der frühere Präsident des Europaparlaments und jetziger SPD-Kanzlerkandidat Martin Schulz ist deutlich weniger zurückhaltend, was ihm in Teilen der polnischen Presse auch sehr übel genommen wird - vornehmlich in denen, die regierungsfreundlich eingestellt sind.

Emmanuel Macron vertritt in diesem Punkt dieselbe Meinung wie Martin Schulz: es kann kein Europa ohne Wertefundament geben, und dieses muss offensiv geschützt werden. So äußerte er sich zur Infragestellung demokratischer Errungenschaften in einem Interview wie folgt: „Europa ist kein Supermarkt, Europa ist eine Schicksalsgemeinschaft" (Wernicke, 2017, S. 3). Die bisher in solchen Fragen eher diskrete französische Regierung stimmt einen deutlich schärferen Ton an, der eine CDU-geführte deutsche Regierung ebenso zu mehr Deutlichkeit veranlassen wird, oder zumindest öfter die unangenehme Frage nach den Konsequenzen einer Verletzung europäischer Grundwerte stellen sollte. 
Die Bedeutung der Werte zeigt sich auch beim Thema Solidarität. Macron sieht die mittel- und osteuropäischen Staaten in der Pflicht, die getroffenen Vereinbarungen zur Umverteilung von Flüchtlingen auch umzusetzen (Brössler et al., 2017, S. 2). Eine „elastische Solidarität", wie sie die polnische Regierung beansprucht, steht damit nicht im Einklang. Als einer der ganz wenigen Präsidentschaftskandidaten, die im Wahlkampf eine flüchtlingsfreundliche Haltung an den Tag gelegt hat, kann Macron diese Position glaubhaft vertreten. Die Flüchtlingskrise hat jedoch Frankreich nicht so akut wie Deutschland betroffen, und ein Flaggschiffprojekt in diesem Bereich ist bislang nicht auszumachen. Es handelt sich also mehr um eine französische Schützenhilfe zur Erreichung des europäisch vereinbarten Status quo, als um eine Möglichkeit der aktiven Neugestaltung.

Ein weiteres Themengebiet, in dem Macron auf Konfrontationskurs mit mittel- und osteuropäischen Mitgliedsländern geht, ist die Reform der Entsenderichtlinie. Bislang können EU-Einwohner aus Ländern mit niedrigeren Arbeitskosten für befristete Arbeitsprojekte in Westeuropa mit besonders wettbewerbsfähigen Löhnen aufwarten. Das ist dem französischen Präsidenten ein Dorn im Auge, denn angesichts einer hohen Arbeitslosigkeit im eigenen Land kann er seinen Wählern nur schwer erklären, warum Arbeitnehmern aus Ländern mit niedrigeren Löhnen der Vorzug gegeben wird (Ducourtieux, 2017, S. 1). Das Problem wird von deutscher Seite aus zwar ähnlich gesehen, der ungleich niedrigere Leidensdruck auf dem Arbeitsmarkt zwang jedoch nicht zu einer harten Linie. Die Mittlerrolle Deutschlands dürfte damit wieder zum Zuge kommen, denn die Bundesrepublik hat kein Interesse an nachhaltig und über Gebühr strapazierten Beziehungen zu seinen östlichen Nachbarn. Die Losung Macrons, für dieselbe Arbeit auf derselben Baustelle denselben Lohn durchzusetzen, wird für konfliktreiche Gespräche sorgen.

\section{Eine soziale Union}

Einer der Gründe, weswegen die EU-Institutionen in beträchtlichen Teilen der europäischen Bevölkerung einen schlechten Ruf genießen, liegt in ihrer begrenzten $\mathrm{Zu}-$ ständigkeit bei populären Themen. Die Bereiche, in denen die EU stark ist, sind eher technokratische Topoi. Mit Handels- und Währungspolitik lässt sich kaum Begeisterung wecken.

Macron will ein „Europa, das schützt“, und räumt damit eine jahrzehntealte französische Position. So wichtig für Deutschland Stabilität ist, so wichtig ist für Frankreich Souveränität. Kein Zufall ist es, dass der Franzose Jean Bodin im 16. Jahrhundert die Komponenten des Souveränitätsbegriffs theoretisch unterfütterte und zu dessen Verankerung als Grundkonzept der politischen Theorie beitrug. Kaum eine wichtige Rede kommt in Frankreich ohne den Verweis auf Souveränität vor, die es zu schützen gelte. Macron stellte sie gleich an den Anfang seiner Rede vor den beiden Parlamentskammern in Versailles am 03. Juli 2017: ,Welches ist dieses Mandat des französischen Volkes? Zuvorderst das Mandat zur Souveränität der Nation, über uns selbst bestimmen zu können“ (Le Monde.fr, 2017, S. 1). Sozialpolitik galt und gilt als entscheidende Domäne staatlichen Handelns. Dass Macron bereit ist, in diesem sensiblen Bereich 
Zuständigkeiten an die EU zu übertragen, darf als großer Fortschritt für die europäische Integration gelten.

Der Aufgabe nationaler Souveränität liegt ein ausgesprochener Pragmatismus zugrunde. Eine soziale Union kann Problemen beikommen, die auf nationaler Ebene kaum noch zu lösen sind. Die EU ist das richtige Instrument, um unerwünschte soziale Folgen der vier Schengener Freiheiten zu vermeiden. Da wo es notwendig war (sprich: dafür alleine zu schwach war), hat sich Frankreich bereits in der Vergangenheit mit der Entwicklung von einem nationalen zu einem europäischen Verständnis von Souveränität angefreundet. Das war zum Beispiel bei der Modernisierungspartnerschaft mit Deutschland in den 1970er Jahren der Fall, als Valéry Giscard d'Estaing einen Rückfall der französischen hinter der deutschen Wirtschaft erkannte und als Antwort eine Vertiefung der wirtschaftspolitischen Instrumente der Europäischen Gemeinschaft erreichte (Woyke, 2010, S. 107ff.).

Die konkrete Ausgestaltung dieser sozialen Union ist noch ziemlich konturlos, man kann sich jedoch darunter von einem europäischen Mindestlohn bis zum Schutz vor Sozialdumping durch höhere Zölle an den EU-Außengrenzen einiges vorstellen. Die Kommission scheint dafür jedenfalls offen zu sein, jüngst schlug sie eine europäische private Altersvorsorge vor, bei der die EU einheitliche Qualitätsstandards für Versicherungsanbieter definieren würde (Wirtschaftswoche, 2017b, S. 1). Eine deutsche Regierung wird sich, welcher Couleur auch immer, diesem Ausbau der EU kaum verweigern können, zumindest solange sie nicht zu Mehrkosten führt. Die Gefahr eines allmählichen Auseinanderfallens der EU, das für die Bundesrepublik verheerende Konsequenzen hätte, ist dafür zu groß.

\section{Europäische Verteidigungsunion}

Die Ungeduld über den bisherigen Stillstand ist bei der Verteidigungspolitik am ehesten zu spüren. Seit dem Scheitern der europäischen Verteidigungsgemeinschaft durch ein Votum des französischen Parlaments im Jahr 1954 sind die Fortschritte bescheiden gewesen. Durch den Brexit eröffnen sich neue Möglichkeiten. Großbritannien hatte sich immer wieder gegen die verteidigungspolitischen Komponenten der EU ausgesprochen, da es ein Aushöhlen der NATO befürchtete und einer vertieften Integration generell ablehnend gegenüber stand.

Im Stillen haben viele Ereignisse gezeigt, dass die meisten EU-Mitgliedsstaaten reif für einen weiteren Schritt zur Verzahnung ihrer Verteidigungsbemühungen sind, wenngleich eine multinationale, europäische Armee weiterhin in weiter Ferne liegt. Seit 2010 sind zum ersten Mal in der Nachkriegsgeschichte deutsche Truppen permanent in Frankreich stationiert. Das Jägerbataillon 291 ist in IllkrichGraffenstaden im Elsass stationiert, und wurde der deutsch-französischen Brigade unterstellt. Entgegen ursprünglicher Befürchtungen gab es keinerlei Proteste oder Bedenken der Bevölkerung gegenüber dieser Stationierung, vielmehr wurden die wirtschaftlichen Vorteile dieser Präsenz wahrgenommen. Dass dies in einem historisch schwer belasteten Gebiet keineswegs eine Selbstverständlichkeit darstellt, mag man sich anhand der Vorstellung ausmalen, welche Reaktionen ein ähnlicher 
Vorgang in einer polnischen Stadt hervorrufen würde. Umso erstaunlicher ist es, dass selbst beim Bundeswehr-Skandal um Nazi-Devotionalien, der seinen Ursprung genau bei jenem Jägerbataillon hatte, französischerseits ebenso unaufgeregt reagiert wurde.

Eine Annäherung zwischen Deutschland und Frankreich wurde zudem durch die deutsche Bereitschaft, auch im militärischen Bereich mehr Verantwortung zu übernehmen, beschleunigt. Eine der ersten Investitionen der Bundeswehr nach der Trendwende im Budget hin zu steigenden statt schrumpfenden Ausgaben im Militärhaushalt bezog sich auf die Flugtransportkapazitäten, die auch Frankreich fehlen. Im April 2017 wurde bekannt, dass bis 2021 eine gemeinsame deutsch-französische Flugstaffel in der Nähe von Paris gebildet werden soll (Wirtschaftswoche, 2017a, S. 1). Die Neuanschaffung von C-130-Hercules-Transportmaschinen durch die Bundeswehr wird durch die Verzahnung mit der Expertise der französischen Luftwaffe, die bereits Erfahrungen mit diesem Modell gesammelt hat, vereinfacht.

Die Konvergenz der europäischen Streitkräfte hat mit dem Konzept der Bundeswehr als „Ankerarmee“ für Einheiten anderer Nationen einen großen Schub bekommen. Einheiten aus den Niederlanden, der Tschechischen Republik und Rumänien bilden zusammen mit der Bundeswehr dauerhaft assoziierte Verbände, die einer gemeinsamen Kommandostruktur unterstellt sind (Bundesregierung, 2017, S. 1). Mit der nun anstehenden Gründung eines europäischen Hauptquartiers, die mit Verspätung das Potenzial des Lissabon-Vertrags ausschöpft, der diese Möglichkeit bereits in Aussicht stellte, dürfte diese Entwicklung weiter vorangetrieben werden. Jetzt muss noch darauf gehofft werden, dass Paris seine langjährige Blockade aufgibt und akzeptiert, dass auch eigene Einheiten theoretisch unter dem Kommando von Offizieren aus anderen Mitgliedsstaaten ihren Dienst versehen. Der Brexit ermöglicht zwar neue Wege, aber diese müssen auch genutzt werden, und dafür sind die Nationen mit den größten Verteidigungsbudgets unentbehrlich.

Neben der Frage der gemischten Einheiten steht auch die der gemeinsamen Rüstungsanschaffungen auf der Agenda. Dies war in der Vergangenheit eine unerschöpfliche Quelle an Konflikten. Die finanziellen Nöte Frankreichs dürften hier ein Ende seines Sonderweges einläuten. In bitterer Erinnerung der europäischen Partner ist der Ausstieg der Republik aus dem Eurofighter-Programm in den 1980er Jahren, der zur Entwicklung des französischen Kampfflugzeugs Rafale führte (Droit, 2010, S. 103), das in nur geringen Stückzahlen produziert wurde und für das zur Amortisierung der Kosten lange Zeit vergeblich Abnehmer im Ausland gesucht wurden.

Die geplante Einrichtung eines europäischen Verteidigungsfonds für Rüstungsausgaben wird Regelungen jenseits von bi- oder multilateralen Projekten ermöglichen. Bisherige Vorhaben wie die gemeinsame Entwicklung eines deutsch-französischen Kampfpanzers, der die französischen Leclerc-Panzer und die deutschen Leopard-Panzer ersetzen wird, ermöglichen eine bessere Interoperabilität der jeweiligen Armeen, ersparen zusätzliche Entwicklungskosten und drücken den Preis bei Wartung und Anschaffung durch Skaleneffekte. Mit dem Verteidigungsfonds öffnet sich die Möglichkeit, weitere europäische Staaten durch einen etablierten Mechanismus einzubeziehen. Langsam löst sich die etablierte Konkurrenz zwischen nationalen Rüstungsunternehmen auf, neben der schon lange bestehenden Unternehmens- 
gruppe Airbus fusionierten 2015 die Branchengrößen Krauss-Maffei Wegmann und Nexter (Höpner, 2015, S. 1).

Schwierigkeiten finden sich neben der erfahrungsgemäß komplizierten Einigung auf die genauen Anforderungen, denen Rüstungsprojekten zu genügen haben, auch bei den Richtlinien zum Waffenexport (Noll, 2016, S. 6f.). Die Bundesrepublik hat eine im internationalen Vergleich restriktive Praxis des Exports, und in der Bevölkerung werden auch die derzeit getätigten Exporte sehr kritisch gesehen. Linke Parteien sind bei diesem Thema besonders aufmerksam und werden zweifelsohne schärfere Einschränkungen beim Export einführen, sollten sie nach der Bundestagswahl gemeinsam eine Regierung bilden. In Frankreich dagegen werden größere Waffenexporte als Erfolg der französischen Industrie gefeiert, daran ändern auch die neuen Mehrheitsverhältnisse nichts. Sofern sich Waffenverkäufe auf NATO-Staaten beschränken, besteht kein Dissens, problematisch wird jedoch jegliches Geschäft mit autoritären Staaten oder Exporte in Krisenregionen.

Ein weiterer Stolperstein bei den Bestrebungen, die Verteidigungspolitik europäisch zu gestalten, wird die Prozedur zum Einsatz von Streitkräften darstellen. In Frankreich wird der Verteidigungsbereich als Prärogative der Exekutive gehandhabt, das Parlament steht in der Regel voll und ganz hinter den Entscheidungen, die der Präsident trifft (Irondelle, Kempin, 2012, S. 118). Ebenso hat die französische Bevölkerung wenig Einwände gegen den Truppeneinsatz im Ausland Die Verfassung der Bundesrepublik sieht in Anbetracht der verheerenden Erfahrungen, die die deutsche Geschichte säumen, eine starke Rolle des Parlaments vor. Der sogenannte Parlamentsvorbehalt sorgt für eine umfassende Kontrolle der Militäreinsätze durch den Bundestag, der diese auch eigenständig verweigern kann. Die deutsche Öffentlichkeit sieht Auslandseinsätze sehr kritisch, entsprechend hitzig sind die darüber stattfindenden Debatten.

Eine vertiefte Kooperation kann damit nur unter der Voraussetzung in Betracht gezogen werden, dass gemischte Einheiten mit deutschen Truppen weiterhin nur unter der Maßgabe eines Parlamentsvorbehalts eingesetzt werden. Gleichzeitig wird Berlin auf Elemente ziviler Krisenprävention in der europäischen Verteidigungspolitik bestehen, die in Frankreich bisher nur in Ansätzen angedacht sind. Besonders bei linken Parteien, aber auch im Mainstream deutscher Politik sind diese kaum wegzudenken.

\section{Neustart mit Fragezeichen}

Im deutsch-französischen Verhältnis ist erkennbar eine neue Dynamik vorhanden. Viele alte Positionen stehen im Rahmen von Kompromissen zu Verfügung, eine neue Ungeduld in der Vertiefung der europäischen Integration ist zu spüren. Macron hat seinen Anteil an diesem Paradigmenwechsel, aber die strukturellen Gegebenheiten helfen ihm dabei außerordentlich. Die aufkommende Flexibilität auf französischer Seite trifft auf eine bis dato nur in engen Grenzen vorhandene Kompromissbereitschaft der deutschen Seite. Wenn die deutsche Blockade bei finanziellen Fragen und die französische Blockade bei Themen, die Auswirkungen auf die nationale Souveränität haben aufgehoben werden, ergibt sich ein einmaliges Zeitfenster der Op- 
portunitäten. Dieses wird sowohl bei einer CDU-geführten, als auch bei einer SPDgeführten Bundesregierung bestehen. Die Analyse hat jedoch gezeigt, dass Macrons Reformwünsche größere Schnittmengen mit linken als mit konservativen Parteien in Deutschland aufweist.

Der Artikel wurde am 03.07.2017 fertiggestellt.

\section{Bibliographie}

Berger A. (2017), Aussichten auf eine Reform der Europäische Union, www.deutschlandfunk.de, 03.07.2017.

Brössler D., Kirchner T., Mühlauer A. (2017), Das Duo Merkel-Macron wirkt bereits, www.sueddeutsche.de, 24.06.2017.

Bundesregierung (2017), Nato: Mehr in Sicherheit investieren, www.bundesregierung.de, 03.07.2017.

CDU, CSU (2017), Für ein Deutschland, in dem wir gut und gerne leben. Regierungsprogramm 2017-2021, www.cdu.de, 03.07.2017.

Cour des comptes (2017), La situation et la perspective des finances publiques. Juin 2017, www. ccomptes.fr, 03.07.2017.

Droit Y. (2010), L'European Fighter Aircraft: le rendez-vous manqué de la coopération aéronautique européenne 1978-1985, "Histoire, Économie Et Société", vol. 29, no. 4, Armand Colin, Paris, S. 103-116.

Ducourtieux C. (2017), Travailleurs détachés: Paris hausse le ton, au risque de braquer les pays de l'Est, www.lemonde.fr, 03.07.2017.

Eurostat (2017), General government deficit/surplus, Code: tec00127, http://ec.europa.eu/eurostat, 03.07.2017

Gougeon J-P. (2012), France-Allemagne: une union menacée? , Armand Colin, Paris.

Höpner A. (2015), Deutsch-französischer Panzerbauer auf Leopardenjagd, www.handelsblatt.com, 03.07.2017.

Irondelle B., Kempin R. (2012), Convergence croissante ou divergence persistante? La coopération franco-allemande en politique, in: 50 ans de relations franco-allemandes, Hrsg. R. Marcowitz, H. Miard-Delacroix, Nouveau Monde Éditions, Paris, S. 111-144.

Lasserre R. (2012), Les relations économiques franco-allemandes: une interdépendance asymétrique, in: 50 ans de relations franco-allemandes, Hrsg. R. Marcowitz, H. Miard-Delacroix, Nouveau Monde Éditions, Paris, S. 145-172.

Lemonde.fr (2017), Congrès à Versailles en direct, www.lemonde.fr, 03.07.2017.

Noll A. (2016), Freunde mit gewissen Vorzügen, www.deutschlandfunk.de, 03.07.2017.

Schmitt O. (2017), Eine neue Außenpolitik für Frankreich? Hollandes Erbe, Macrons Herausforderungen, "WeltTrends" 129 / Juli, S. 39-43.

SPD (2017), Es ist Zeit für mehr Gerechtigkeit: Zukunft sichern, Europa stärken. Das Regierungsprogramm 2017 bis 2021, www.spd.de, 03.07.2017

Uterwedde H. (2017), Frankreichs Wirtschaft: Reformbedarf und Potenziale, "WeltTrends" 129 / Juli, S. 34-38.

Wernert Y. (2017), Reform um jeden Preis. Ein Blick auf die Präsidentschaftswahlen in Frankreich, "WeltTrends" 125 / März, S. 11-14.

Wernicke C. (2017), Der Anfang einer Renaissance, www.sueddeutsche.de, 24.06.2017. 
Wirtschaftswoche (2017a), Bundeswehr will mit Frankreich Fliegerstaffel betreiben, www.wiwo. de, 29.06.2017.

Wirtschaftswoche (2017b), EU stellt europäische Altersvorsorge Pepp vor, www.wiwo.de, 29.06.2017.

Woyke W. (2010), Die Außenpolitik Frankreichs, VS Verlag für Sozialwissenschaften, Wiesbaden.

The resurgence of the Franco-German partnership.

A review in the double election year 2017

\section{Summary}

This article argues that the relations between France and Germany have gained a new dynamic since the French elections of 2017. While president Macron shares more common views with the German left, he will most likely accommodate with conservative parties as well. European reforms have to be expected in the fields of finance, social and defence policy.

Key words: France, Germany, European Financial Policy, European Social Policy, European Defence Policy

\section{Streszczenie}

W artykule zwrócono uwagę, że relacje pomiędzy Francją i Niemcami nabrały dynamiki po wyborach prezydenckich we Francji w 2017 r. Jakkolwiek Macron miał więcej powiązań z niemiecką lewica, potrafi również znaleźć wspólny język z partiami konserwatywnymi w Niemczech. Należy spodziewać się w UE reform w obszarze finansów, polityki społecznej i obrony.

Slowa kluczowe: Francja, Niemcy, europejska polityka finansowa, europejska polityka społeczna, europejska polityka obrony 
